\title{
Results of 300 cases of mini - percutaneous nephrolithotomy at the Thanh Hoa General Hospital in Vietnam
}

\author{
S.L. Van, T.T. Thanh, H.L. Dinh and N.L.D. Phuong \\ Department of Urology of Thanh Hoa General Hospital, Vietnam \\ Corresponding author: T.T. Thanh \\ E-mail: tungtnqy@gmail.com \\ Genet. Mol. Res. 20 (1): gmr18746 \\ Received December 30, 2019 \\ Accepted April 20, 2020 \\ Final Revision January 25, 2021 \\ Published March 30, 2021 \\ DOI http://dx.doi.org/10.4238/gmr18746
}

\begin{abstract}
We evaluated the results of mini-percutaneous nephrolithotomy (mini-PCNL) under ultrasonic guidance for treatment of kidney stones at Thanh Hoa General Hospital. This descriptive study examined, from July 2016 to September 2018, 300 cases of kidney stones performed mini-PCNL under ultrasonic guidance. Nephrolithotripsy was done by 30 and $100 \mathrm{w}$ Homium lasers. The 300 patients included $63.3 \%$ men and $36.7 \%$ women. The mean age was $42.13 \pm 33.4$ years. Recurrent stones were found in $15 \%$, renal pelvis and one calyx stone in $51.7 \%$. Hydronephrosis before surgery at level 1 was found in $46.7 \%$. Stone average length was $3.11 \pm 0.63 \mathrm{~cm}$ and average area was $5.16 \pm 2.41 \mathrm{~cm}^{2}$. Tunnel position in the middle calyx was found in $68.3 \%$. Average surgery time averaged $58.42 \pm 27.8$ minutes. Bleeding during surgery was reported for 15 patients, hematuria after surgery in two patients, and fever after surgery in 46 patients. The average hospitalization duration was $4.6 \pm 2.3$ days. Early stones clearance rate was $78.3 \%$. Mini-PCNL under ultrasound guidance with a $30-100 \mathrm{w}$ Holmium Laser energy source is a useful method of treating kidney stones safely, achieving an early stone clearance efficiency of $82.4 \%$,and a low rate of postoperative complications of $16.7 \%$. This method can effectively replace open surgery for the treatment of kidney stones.
\end{abstract}

Key words: Nephrolithiasis; Nephrolithotripsy; Renal stone; PCNL; Holmium Laser 


\section{INTRODUCTION}

Urinary stone disease influences all age groups. The reported prevalence rate of stone disease is $5-12 \%$ in men and $4 \%-7 \%$ in women. Stone formation is affected by gender, age and geography. Men's possibility of forming stones is more than women's (Mehmet and Ender, 2015).

Over the years, there has been a great advancement in technology for minimally invasive treatment of urinary stones. Percutaneous nephrolithotripsy (PCNL) is currently the most popular minimally invasive method of treating renal stones. The use of PCNL has steadily increased as a replacement for the traditional open surgery besides other methods such as Retrograde IntraRenal Surgery (RIRS) and Extracorporeal (ESWL). RIRS was not recommended as a first-line treatment for renal calculi until 2013 according to the EAU guidelines. In recent years, significant improvements have been made to the PCNL method in terms of identifying stone location, puncture of the renal pelvis and calices, miniaturization of the access tracts, and enhancing instruments for the endoscopic grinding of stones, making it a highly efficient and much safer way of treating renal stones. From 2008, mini-PCNL has been performed worldwide with a smaller access tract to the kidney that measured only $12-20 \mathrm{Fr}$, resulting in higher stone-free rates (SFR) and less complications than the standard PCNL which uses larger tracts of $24-30 \mathrm{Fr}$ (Nagele et al., 2008; Hiep Nguyen Trong Hoang, 2016).

In Vietnam, the standard PCNL was performed in 2004 and has been carried out in many hospitals till now. However, the standard fluoroscopy-guided PCNL has many limitations and undesirable effects. On the other hand, the new mini-PCNL has been implemented only in a few hospitals in the country since 2012. Therefore, we conducted this study to evaluate the feasibility and effectiveness of mini-PCNL in the treatment of renal stones with a puncture technique and creates percutaneous tunnel under ultrasound guidance and uses lithotripsy energy by $30-100$ w Holmium laser.

\section{MATERIAL AND METHODS}

\section{Research subjects}

A total of 300 patients diagnosed with kidney stones received mini-PCNL treatment from July 1, 2016 to August 1, 2017 at the Department of Urology - Thanh Hoa General Hospital. Indications for lithotripsy include:

Solitary stones of the renal pelvis (S1-2), partial or complete staghorn stones (S3-45) according to the classification of Moores (1976).

Caliceal stones or calyx diverticular stones. Pelvis - combined pelvic and ureteral stones or stones of the upper ureter that had been treated by a failed prior retrograde lithotripsy. Kidney stones has been unsuccessfully treated in lithotripsy; kidney stones have recurred after surgery.

All the patients agreed to participate in the experiment and do not deny the results of the experiment to be provided in the research paper. All procedures followed were in accordance with the ethical standards of the institutional committee on human experimentation and with the Helsinki Declaration of 1975, as revised in 2000. 


\section{Research methodology: cross-sectional description}

The research period was from July 2016 to September 2018. Patients were included and diagnosed to evaluate morphology, bilateral kidney function, anatomical correlation of renal calyx and pelvis with morphology, location of stones, and identify the accompanying kidney pathology. Patients were actively treated for urinary tract infection before surgery.

Prepare equipment and instruments: urological endoscope apparatus, Hitachi- Ezu ultrasound machine with Convex 3-5 MHz transducer. Holmium Laser Sphin X 30W-100W lithotripsy machine with 550 um canopy heads. Karl Storz water pump with speed of 100 $800 \mathrm{~mL} / \mathrm{min}$, rigid uretero-renoscope size 6.2/6.5Fr. Amplatz renal dilation set $6-18 \mathrm{Fr}$ and sheath 18Fr made of Seplou plastic.

The procedure was as follows:

General anesthesia.

The patient lies on his back, the doctor cystoscopically evaluates the condition of the ureter and places the catheter on the renal pelvis. If the ureter is narrow and cannot fold the guide wire, do not continue mini-PCNL.

Move the patient to a lying on the opposite side with a cushion of lumbar pits. Ultrasound to check the position and morphology of pyelonephritis of the kidney, determine the most convenient renal calyx to access, has the shortest distance to the skin, access to other kidney stations.

Skin incision, abdominal wall of waist area $0.7 \mathrm{~cm}$ at the puncture site is determined in accordance with the ultrasound scan. Insert the 18-gauge needle into kidney under ultrasound guidance. When you enter the renal calyxes, there will be signs of a needle touching the stone or a urine.

Put the curved lead wire into the renal pelvis. Create tunnel with Amplatz renal dilation set $6-18 \mathrm{Fr}$. Place a sheath $18 \mathrm{Fr}$ into tunnel to the renal pelvis and calyx. Using a ureteroscope to determine the position, number and size of stones related to the structure and morphology of the renal pelvis and calyx.

Dissolve kidney stones with energy Holmium Laser $30 \mathrm{~W}$ and $100 \mathrm{~W}$ into small pieces, combine with water pump by machine with the pressure of $200-400 \mathrm{~mL} / \mathrm{min}$. Pump to remove gravel or use pincers. Check for calculi. Remove the ureter catheter and place the JJ catheter downstream or upstream. Renal drainage with sonde Mono J 14-16Fr through the tunnel.

Monitoring after procedure: Abdominal condition, general condition, quantity and color of urine through renal drainage and urethral catheter. Early detection of complications such as bleeding and urinary tract infections.

X-ray and ultrasound examination after 3 days to determine the SFR without gravel $>4 \mathrm{~mm}$. Renal drainage and urethral catheter withdraw after 2-6 days and patients discharged. Re-examination after 15-30 days, X-ray and ultrasound examination to determine the SFR and indicate JJ withdrawal.

\section{RESULTS}

The 300 patients included 190 men (63.3\%) and 110 women (36.7\%). Average age was $42.13 \pm 33.4$ years (range: 22 - 84 years). The most aged 41 - 60 years old. There were 15 patients who had ESWL and stones were located in the kidney. 24 patients had 
undergone a prior open surgery to remove their renal and ureteral stones (13.3\%): 5 patients $(1.67 \%)$ patients had undergone a previous retroperitoneal laparoscopic surgery to treat ureteral stones on the same side.

Of the 300 patients, $21.7 \%$ had a medical history including:

15 cardiovascular patients, hypertension;

10 patients with chronic heart disease;

10 diabetics; 5 patients with first stage renal failure;

5 patients with scoliosis.

There were 20 patients with urological infection treated before surgery.

The left stone has 175 patients, accounting for $58.3 \%$ and the right stone $41.7 \%$. Average length of gravel: $3.1 \pm 0.6 \mathrm{~cm}(1-4.9 \mathrm{~cm})$, average width: $2.2 \pm 0.3 \mathrm{~cm}(1-4.2$ $\mathrm{cm})$ and average area of gravel $5.1 \pm 2.4 \mathrm{~cm}^{2}\left(1-16.72 \mathrm{~cm}^{2}\right)$.

Table 1. Renal stone characteristics of the 300 patients.

\begin{tabular}{llc}
\hline Morphology and location of stones & No. of patients & $\mathbf{\%}$ \\
\hline S2 (renal pelvis) & 65 & 21.7 \\
S3 (renal pelvis + calyce) & 155 & 51.7 \\
S4 (renal pelvis + calyces) & 45 & 15 \\
S5 (renal pelvis + 3 calyces) & 10 & 3.3 \\
Lower calyce & 10 & 8.3 \\
Total & 300 & 100 \\
\hline
\end{tabular}

First-degree hydration was most common with 140 patients (46.7\%). Grade II 75 patients, accounted for $25 \%$. Grade III 20 patients, accounting for $6.6 \%$ and non- dilated pyelonephritis in 65 patients, accounting for $21.7 \%$.

All patients were anesthetized and put on recumbent position.

Choosing renal calyx to access, create a tunnel: The middle calyxes group is the most chosen $68.3 \%$, the upper calyxes group is the lowest chosen $6.7 \%$ and the lower calyxes group is $25 \%$. All punctures into renal calyx and create a tunnel under ultrasound guidance were successful. The percentage of creating one tunnel accounted for the majority of 290 patients (98.3\%) and creating two tunnels in five patients accounting for $1.7 \%$ due to complicated S5 gravel having branches in a separate calyx. Puncture into pyelo-calyxes system is the majority 215 patients, accounting for $71.7 \%$. Direct puncture in stones 85 patients $(28.3 \%)$.

Table 2. Localization of kidney stones in the 300 patients.

\begin{tabular}{lllllll}
\hline $\begin{array}{l}\text { Renal } \\
\text { calyces }\end{array}$ & S2 stone & S3 stone & S4 stone & S5 stone & $\begin{array}{l}\text { Lower } \\
\text { calyce stone }\end{array}$ & Total \\
\hline Upper & 5 & 15 & 0 & 0 & 0 & $20(6.7 \%)$ \\
Middle & 30 & 120 & 40 & 10 & 5 & $205(68.3 \%)$ \\
Lower & 30 & 20 & 5 & 0 & 20 & $75(25 \%)$ \\
Total & 65 & 155 & 45 & 10 & 25 & $300-100 \%$ \\
\hline
\end{tabular}

Happening in surgery: 15 patients $(5 \%)$ bleeding during surgery from the tunnel location in the S2 - S5 stone does not cause hydronephrosis, thick renal parenchyma, no hemodynamic disorders and no blood transfusion. 
Table 3. Operative time for the 300 kidney stone patients.

\begin{tabular}{|c|c|c|c|c|c|}
\hline Times & S2 stone & S3 stone & S4 stone & S5 stone & $\begin{array}{l}\text { Lower calyce } \\
\text { stone }\end{array}$ \\
\hline Tract dilation & $6.41 \pm 5.91$ & $7.45 \pm 4.50$ & $7.72 \pm 1.35$ & $8.31 \pm 3.11$ & $5.97 \pm 1.21$ \\
\hline Lithotripsy & $41.9 \pm 6.31$ & $52.46 \pm 1.14$ & $70.43 \pm 7.49$ & $91.52 \pm 2.35$ & $53.47 \pm 6.41$ \\
\hline Sonde JJ & $4.32 \pm 2.61$ & $4.47 \pm 2.13$ & $3.82 \pm 2.26$ & $4.58 \pm 4.61$ & $4.66 \pm 2.22$ \\
\hline
\end{tabular}

Average operating time: $58.42 \pm 27.8(32-110 \mathrm{~min})$, the longest in the stone group S5, group S2 and renal calyx has the shortest time, the catheter JJ 6 or $7 \mathrm{Fr}$ is placed downstream. Benefits in 290/300 patients accounted for 96.7\%. Six patients $(2 \%)$ placed JJ upstream due to the tunnel in the lower channel perpendicular to the pyelo-ureter axis and four patients did not place $\mathrm{JJ}$.

Long operative time increases the duration of anesthesia and may risk postoperative pulmonary complications. Also it increases blood loss, the need for transfusion and overall complication rates. In addition, a short operative time is important because it is cost effective.

Renal drainage by catheter Mono $\mathrm{J} 14 \mathrm{Fr}$ in 48 patients $(80 \%), 16 \mathrm{~F}$ in 12 patients $(20 \%)$.

Happening after surgery: Complications after surgery in 49 patients accounting for 16.3\% including: 46 patients with fever and stable treatment. There are 2 patients with hematuria need to intervene renal vessels $(0.67 \%$ and 1 patient with S4 gravel due to the tip of $\mathrm{JJ}$ catheter not coming to the bladder after Mono $\mathrm{J}$ withdrawal with small round fluid around the kidney, no need to aspire for internal fluid, only retrograde endoscopy to pull the tip of JJ down to the bladder on day 3).

Average duration of hospitalization: $5.6 \pm 2.3$ days ( $3-17$ days). Renal drainage is withdrawn after 2 - 5 days $(2.1 \pm 1.2$ days $)$. X-ray and ultrasound examination on day 3 after surgery had $247 / 300$ patients still having stones $>4 \mathrm{~mm}$, the early SFR after surgery $82.4 \%$. Postoperative analgesia is administered for 2 days.

Table 4. Stone free rate after PCNL.

\begin{tabular}{|c|c|c|c|c|c|}
\hline Stone free rate & S2 stone & S3 stone & S4 stone & S5 stone & $\begin{array}{l}\text { Lower calyce } \\
\text { stone }\end{array}$ \\
\hline After procedure & $86.2 \%$ & $81.7 \%$ & $71.2 \%$ & $68.7 \%$ & $100 \%$ \\
\hline After 1 month of PCNL & $94.1 \%$ & $88.4 \%$ & $77.8 \%$ & $76.7 \%$ & $100 \%$ \\
\hline
\end{tabular}

Distal postoperative monitoring: 300 patients were re-examined after 15 - 30 days. Painless, no fever and X-ray and ultrasound examination showed 266/300 patients with the SFR (stones $<4 \mathrm{~mm}$ ) reached $88.7 \%$ and JJ was withdrawn. There were 34 patients with stones $>4 \mathrm{~mm}(11.3 \%)$. Indicate ESWL for 18 patients, 11 patients after the JJ withdrawal had stones to the ureter and had retrograde endoscopy to lithotripsy by laser energy.

\section{DISCUSSION}

In the treatment of kidney stones so far, there have been many great developments with the use of less invasive intervention techniques instead of open surgery. Standard 
PCNL with a 24-30Fr tract size has been widely recognized as one of the most powerful instruments as it allows for rapid stone removal and is highly efficient in treating large kidney stones and complex staghorn stones.

A number of studies showed that PCNL can be performed successfully without risk of complications in patients with a history of previous open surgery or PCNL. Especially with the mini-PCNL which uses smaller access tract (18Fr), the complications of bleeding and kidney parenchyma have been reduced. The use of high power $80 \mathrm{w}$ Homium laser enables a faster destruction of stones and enhances stone fragmentation. Stone fragments can be easily removed during the lithotripsy procedure, resulting in a higher efficiency rate of stone clearance. In the treatment of complex staghorn stones where multiple accesses are needed, the use of smaller tracts has demonstrated MiniPCNL's superiority over other methods. Some studies have also reported that Mini-PCNL was as efficient as the standard PCNL in the treatment of larger kidney stones. This technique can use a $7.5 \mathrm{Fr}$ ureter endoscope instead of a specialized kidney endoscope to flexibly manipulate into the calyxes of kidney, especially prevailing when the calyx neck is narrow. Use Holmium Laser 100W energy to dissolve stones quickly, safely, improve stone cleanliness, reduce kidney damage, reduce the risk of bleeding and reduce reflux pressure. Thanh Hoa General Hospital has started to implement mini-PCNL application under ultrasound guidance to treat kidney stones. Over 300 cases were performed showing the SFR is $82.4 \%$. When compared with a number of authors doing internal and external, no statistically significant differences were found between these ratios: Hoang Long (2017), mini- PCNL study at Hanoi Medical University Hospital showed a 77.7\% cleanliness rate (Hiep Nguyen Trong Hoang, 2016). According to Abdelhafez (2012), the SFR is $83 \%$.

Ultrasonic tunneling was proposed in 1976. In recent years, ultrasound guided access during percutaneous nephrolithotomy has been widely reported as an approach with high success rate. Gamal et al., (2011) reported a series of 34 PCNL in which only ultrasound guidance was used during the whole procedure with the SFR 94\%. We determine the location of stones and treat them completely under ultrasound guidance. Till now, there has been little domestic research on ultrasonic guidance.

Ultrasonic guidance has shown many convenient and accurate advantages, showing clear stone or no visible contrast on X-ray. Despite the many advantages of this method, research on ultrasound localization of kidney stones has been relatively limited in Vietnam. Poking under the ultrasound guidance is making an approach in the direction of the ultrasound layer to give the most visible image and the most favorable puncture so that the landmark can be determined to be in the dilated renal pelvis or directly into the gravel. Therefore, the puncture position will not be fixed. The ultrasound assessment is related to the kidneys with stones and other internal organs, showing the thickness and texture of the abdominal wall soft tissue that the needle pokes through to avoid organ damage. Ultrasound can reveal gas-filled images of the colon sometimes lying behind the kidneys, especially in patients undergoing surgery. However, ultrasound positioning should clearly show the path and the puncture needle to stop in the renal pelvis or when touching stones to avoid the risk of the needle penetrates the neck or the adjacent renal parenchyma causing bleeding.

Observing the needle tip will definitely determine the depth and direction of the hot tool, safe operation, high success rate, different from under-X-ray probe, which takes a lot of time, uses contrast dye and at risk of radiation. Under the ultrasound guidance, the location of the gravel branches in the renal pelvis group was measured in front or back plane 
so that the needle could be oriented and the tunnel into the optimal kidney channel from which the lens can be removed. The calyx of kidney do not damage the neck. Ultrasound during and after surgery clear stones, detect complications during surgery, significantly reducing operative time. Using a color ultrasound can prevent damage to blood vessels when puncturing through the parenchyma.

Other advantages of ultrasound guidance over fluoroscopic guidance include radiation avoidance, and shorter operative time. Ultrasound can be used both intraoperatively and post-operatively to verify stone clearance, which significantly reduces operative times.

\section{The position of the patient in performing PCNL}

The prone position is considered to have an effect on resuscitation anesthesia, especially in patients with cardiovascular and respiratory diseases, or obesity. Recent studies have conducted in an inclined position for patients to reduce the risk of respiratory and circulation. We have chosen the inclined position, the inclination based on many different factors. The inclined posture reduces the risk of respiratory, circulatory and well monitoring of patients during surgery, so it is possible to detect complications of visceral injury or drainage into the abdomen and retroperitoneal (Osman et al., 2005; Cheng et al., 2010). The puncture site creates a tunnel that usually enters the free side or slopes backward so that the renal parenchyma is thicker but minimizes the risk of bleeding (Ichaoui et al., 2019).

\section{Choosing the location of renal calyxes to make a tunnel into the kidney}

The most important step is the decision to success and the complication of intervention. Selecting the way to the renal pelvis or directly into the stone based on preimaging and ultrasound imaging during surgery. The evaluation of the type of kidney stone on CT scan and ultrasound scan is very necessary for determining the most appropriate entrance before puncture (Basiri et al., 2016; Gunawan et al., 2019).

Staghorn stones are mostly treated by percutaneous nephrolithotomy (PCNL), either with an upper-pole (UP) or non-upper (lower- or middle-) pole (NP) approach (Knoll, 2019). NP access has a lower risk of bleeding and thoracic complications but may not be sufficient for complete stone clearance. UP access is advocated as the preferred approach, because of direct access to the collecting system. However, it is associated with a higher complications rate, including pneumothorax and hydrothorax, and a higher risk of bleeding (Rizvi et al., 2017; Jones et al., 2018).

Puncturing into the renal pelvis accounts for the majority of $71.7 \%$ and poking directly into the stones is $38.3 \%$ (indicated for complex stone or stones in separate renal calyx do not dilate). In fact, the pelvis and its dependent middle calyx offer the largest area for renal puncture, and from this site of Amplatz placement access to the other calices might be more favorable and easier. Similarly, access to the ureter to assess its patency and to place the Double J stent was also easier (Van Can et al., 2019).

The location of puncture and tunneling into the lower calyxes in 75 patients accounting for $25 \%$ is done when the access road to the lower stones is directly needed. The lowest rate of puncture on the upper calyxes was 20 patients $(6.7 \%)$ selected for pyelo stone 
plus the upper stone. This position is more difficult, entangled ribs, associated pleura, difficult manipulation when lithotripsy and observing other renal calyxes (Reddy and Shaik, 2016).

\section{Percutaneous renal lithotripsy technique}

Poking out urine is the most sure sign of having entered the renal pelvis. For patients with an old incision in the lumbar flank, favorable probe placement in the recumbent position because the kidneys are relatively fixed due to old surgical adhesive. Our study found 45 patients with recurrent gravel disease, including 40 patients with old lumbar surgery, five patients with retroperitoneal laparoscopy. Evaluation of surgery showed that the organization around the kidney and parenchyma of the kidneys was stiffer, when puncturing and dilating the tunnel into the renal pelvis is more difficult.

The rate of stone cleanup is not only related to the size of the main stone, but also greatly dependent on the number of small stones attached. We only calculate the size of the main stone, so for SSH with many separate small stones in the stations, the removal of the gravel after the canopy will take longer and be more thorough. The advantage of Amplatz 18Fr plastic is that it is thin and soft, so it can be inserted deep and spleen through the kidney stations, sometimes even angled Amplatz corner to have access to gravel in small stations and removed by using water pump or gravel pliers directly. However, for small, narrow stands, the movement to take Amplatz through the neck should be careful not to hurt the neck causing bleeding and this is also difficult to remove the stones.

Difficulty is encountered when the gravel is located in the adjacent station, parallel to the access position. The gravestone-antiquated citadel is then opposite to the direction of the Amplatz so it is difficult to access the gravel so that it can be easily spread and left in separate stations.

The time taken to probe, create a tunnel and place the Amplatz on the pyelonephritis depends on many factors such as: Experience of the surgeon, the size of the stone is the target for direct puncture and the degree of dilatation of the kidney. If the stone is located in the renal pelvis, then the exploration and spread of the stone will likely be difficult. If the stone is located in the kidney, it is best to poke the stone directly, and then the Amplatz will come into direct contact with the gravel, so when the stones are small, the gravel will be sucked out and avoid the introduction of Amplatz through the neck. at risk of tearing the neck causing bleeding. The more the kidneys relax, the easier it will be to prick and place the Amplatz. Grade II hydronephrosis to see $25 \%$ will be most convenient when probing, placing Amplatz and lithotripsy. The first degree of hydronephrosis was found in $46.7 \%$, and $21.7 \%$ were not hydrated. If the stones are large and the renal pelvis is not dilated, the thick kidney parenchyma will have difficulty in tunneling. An easy poke and tunnel into the kidney does not mean lithotripsy is also favorable. Dilated to the third level of water retention of $9.3 \%$ will cause difficulty lithotripsy due to moving stones, prolonging surgery time, high risk of missing stones, longer time for renal drainage.

Recent studies (Bum Soo Kim, 2015) have shown that shrinking tunnel and renal tubular dimensions is the gold standard to increase the efficiency of lithotripsy and reduce complications of standard PCNL. The first mini-PCNL and micro-PCNL are used for children and are also highly effective and safe for use in adults mainly with the $18 \mathrm{Fr}$ tunnel. In order to minimize tunnel size and further reduce complications, Desai and Solanki (2011) 
described the MicroPerc technique with the concept "All seeing needle" as a 1-step "Single step PCNL" method using a scope. $0.9 \mathrm{~mm}$ Fr small through the tunnel $4.85 \mathrm{Fr}$, laser fragments of gravel are taken through a vacuum and not using a gravel removal device. In 2013, Desai described the Ultra mini PCNL (UMP) technique using a 6 Fr tube through the 11 - 13Fr tunnel with a high rate of $88.9 \%$ gravel removal and fewer complications. However, both methods specify lithotripsy of less than $2 \mathrm{~cm}$ in the subcellular kidney.

The rate of clean gravel removal after ablation was reported to be from 62 to $94.3 \%$, a large difference between the authors due to heterogeneity in gravel selection criteria for the implementation of energy efficiency as well as methods and energy sources. gravel canopy. The rate of stone removal depends on many factors such as the size of the stone, the number of stones, the degree of complexity, the location of the stones, the pathological characteristics of the kidneys and especially the surgical skills and the method used. Our $\mathrm{NC}$ had a gravel ratio of $11.3 \%$ related to SSH S4 - S5 complex, which has many stones in separate kidney stations so it is very difficult to access them all (Xiao et al 2017).

But does the standard patient benefit from miniaturized PCNL? This answer cannot be given yet, and no guideline gives advice on this. But what can be observed in daily practice is an increasing frequency of PCNLs in medium-sized stones instead of ureteroscopy or extracorporeal shock wave lithotripsy, justified by miniaturization. Caution is advisable considering the very weak available data supporting this concept. It is worldwide accepted, that retrograde ureteroscopy, that competes with mini- PCNL, comes with significantly lower complication risk than percutaneous surgery. Maxi complications may occur even with micro access. Size might matter, but what matters more are the right indication and a skilled surgeon (Mehmet and Ender, 2015; Fahmy et al., 2017).

\section{Disadvantages of Mini-PCNL}

Use of Mini-PCNL equipment leads to longer operative times to achieve fragments of a size small enough to fit through the smaller instrument channel. Furthermore, m-PCNL does not allow for stone extraction but relies on passive clearance of fragments similar to SWL. In this respect, stone analysis can be difficult (unless sieved by the patient during urination). This inability to retrieve fragments leads to a higher rate of steinstrasse, therefore necessitating urgent drainage of the collecting system via JJ stenting.

To improve the safety of PCNL, there has been a trend towards using progressively smaller nephrostomy tracts. The modified techniques of PCNL, such as miniperc, microperc, and ultraminiperc, have been introduced for clinical use with the aim to reduce the likelihood of major complications, such as bleeding and renal injury that could enable accomplishing these procedures in an outpatient setting. There is an increasing trend towards outpatient PCNL, which represents a safe and feasible surgical option for carefully selected patients. Our present results are comparable to the growing body of evidence suggesting that the outpatient PCNL procedure can be safely done with excellent outcomes, and could potentially become the standard of care for many patients

The changes in PCNL techniques included not only decrease of the working instruments diameter but also improvement of patient positioning, safer and more accurate tract creation techniques, new imaging modalities, evolvement of intracorporeal lithotripters and incorporation of flexible instruments for efficient collecting system screening (Sabler et al., 2018). 


\section{CONCLUSIONS}

Percutaneous lithotripsy small tunnel 18Fr under ultrasound guidance and energy source $30-100 \mathrm{w}$ Holmium laser lithotripsy is a safe treatment for kidney stones and has a high early $82.4 \%$ cleanliness efficiency and complication rate. After surgery, $16.7 \%$ is consistent with the characteristics of stone disease in Vietnam. This method has replaced open surgery in the treatment of large and complex kidney stones. This is a new option for the treatment of kidney stones, and it is possible to do it routinely in our country with the existing equipment conditions.

\section{ACKNOWLEDGMENTS}

The authors would like to thank the Department of Urology of Thanh Hoa General Hospital for the support.

\section{CONFLICTS OF INTEREST}

The authors declare no conflict of interest.

\section{REFERENCES}

Abdelhafez MF, Bedke J, Amend B, El Ganainy E, et al (2012). Minimally invasive percutaneous nephrolitholapaxy $(\mathrm{PCNL})$ as an effective and safe procedure for large renal stones. BJU Int. 110: e1022-e1026.

Basiri A, Kashi AH, Zeinali M, Nasiri M, et al., (2016). Ultrasound - guided access during percutaneous nephrolithotomy: entering desired calyx with appropriate entry site and angle. Int. Braz. J. Urol. 42(6): 1160-1167.

Bum SK (2015). Recent advancement or less invasive treatment of percutaneous nephrolithotomy. Korean J. Urol. 56: 614-623.

Cheng F, Yu W, Zhang X, Yang S, et al. (2010) Minimally invasive tract in percutaneous nephrolithotomy for renal stones. J. Endourol. 24(8): 1579-1582.

Desai J and Solanki R (2013). Ultra-mini percutaneous nephrolithotomy (UMP): one more armamentarium. BJU Int. 12(7): 1046-1049.

Fahmy A, Rhashad H, Algebaly O and Sameh W (2017). Can percutaneous nephrolithotomy be performed as an outpatient procedure? Arab J. Urol. 15(1): 1-6.

Gamal WM, Hussein M, Aldahshoury M, Hammady A, et al. (2011). Solo ultrasonography-guided percutaneous nephrolithotomy for single stone pelvis. J. Endourol. 25(4): 593-596.

Gunawan S, Birowo P, Rasyid N and Atmoko W (2019). Outcome and safety of upper pole versus non-upper pole single puncture PCNL for staghorn stones: a systematic review and meta-analysis. F1000Res. 8: 537.

Hiep NTH (2016). Tán sỏi qua da đường hầm nhỏ - mini PCNL. Sỏi Tiết Niệu . Retrieved from https://www.soitietnieu.com/tan-soi-qua-da-duong-ham-nho-mini-penl.html.

Ichaoui H, Samet A, Hermi A, Hedhli H, et al. (2019). Percutaneous Nephrolithotomy (PCNL): Standard Technique Versus Tubeless-125 Procedures. Cureus. 11(3): e4251-e4251.

Jones P, Elmussareh M, Aboumarzouk OM, Mucksavage P, et al. (2018). Role of minimally invasive (micro and ultramini) PCNL for adult urinary stone disease in the modern era: evidence from a systematic review. Curr. Urol. Rep. 19(4): 1-8.

Knoll T (2019). Is mini-percutaneous nephrolithotomy the way to go for renal stones? No! Curr. Op. Urol. 29(3): 312313.

Mehmet NM and Ender O (2015). Effect of urinary stone disease and its treatment on renal function. W. J. Nephrol. 4(2): 271-276.

Moores WK (1976). The surgical significance of the proteus stone. Br. J. Urol. 48(6): 399-401. doi: 10.1111/j.1464410x.1976.tb06666.x.

Nagele U, Schilling D, Anastasiadis AG, Walcher U, et al. (2008). Minimal-invasive perkutane Nephrolitholapaxie (MIP). Urologe A. 47(9): 1066-1073.

Osman M, Wendt-Nordahl G, Heger K, Michel MS, Alken P, et al (2005). Percutaneous nephrolithotomy with ultrasonography- guided renal access: experience from over 300 cases. BJU Int. 96(6): 875-878. 
Reddy SV and Shaik AB (2016). Outcome and complications of percutaneous nephrolithotomy as primary versus secondary procedure for renal calculi. Int. Braz. J. Urol. 42(2): 262-269.

Rizvi SAH, Hussain M, Askari SH, Hashmi A, et al. (2017). Surgical outcomes of percutaneous nephrolithotomy in 3402 patients and results of stone analysis in 1559 patients. BJU Int. 120(5): 702-709.

Sabler IM, Katafigiotis I, Gofrit ON and Duvdevani M (2018). Present indications and techniques of percutaneous nephrolithotomy: What the future holds? Asian J. Urol. 5(4): 287-294.

Van Can T, Phuc VDH and Son NH (2019). The surgical outcome of percutaneous nephrolithotomy based on principle of right triangle for renal access with fix C-arm and no the contrast. Biomed. J. Sci. Tech. Res. 17(5): 13099-13102.

Xiao Y, Li D, Chen L, Xu Y, et al. (2017). The RIRS scoring system: An innovative scoring system for predicting stonefree rate following retrograde intrarenal surgery. BMC Urol. 17(1): 1-8. 